\title{
BMJ Open Trial protocol: preoperative administration of tranexamic acid in sleeve gastrectomy (PATAS) to reduce haemorrhage rates. A randomised controlled trial
}

\author{
Marjolijn Leeman (D) , J Jeannine Huisbrink, ${ }^{2}$ Julie M A Wijnand, ${ }^{1}$ L Ulas Biter, ${ }^{1}$ \\ Serge J C Verbrugge, ${ }^{3}$ Martin Dunkelgrun, ${ }^{1}$ Jan A Apers ${ }^{1}$
}

To cite: Leeman M, Huisbrink J, Wijnand JMA, et al. Trial protocol: preoperative administration of tranexamic acid in sleeve gastrectomy (PATAS) to reduce haemorrhage rates. A randomised controlled trial. BMJ Open 2020;10:e034572. doi:10.1136/ bmjopen-2019-034572

- Prepublication history for this paper is available online. To view these files, please visit the journal online (http://dx.doi. org/10.1136/bmjopen-2019034572).

Received 25 September 2019 Revised 04 December 2019 Accepted 21 January 2020

Check for updates

(c) Author(s) (or their employer(s)) 2020. Re-use permitted under CC BY-NC. No commercial re-use. See rights and permissions. Published by BMJ.

${ }^{1}$ Surgery, Franciscus Gasthuis en Vlietland, Rotterdam, The Netherlands

${ }^{2}$ Pharmacology, Franciscus Gasthuis en Vlietland, Rotterdam, The Netherlands

${ }^{3}$ Anaesthesiology, Franciscus Gasthuis en Vlietland, Rotterdam, The Netherlands

Correspondence to

Marjolijn Leeman

m.leeman@franciscus.nl

\section{ABSTRACT}

Introduction Fast-track protocols often include shortterm thromboprophylaxis and short length of hospital stay. These treatment strategies may negatively affect the occurrence and diagnosis of postoperative haemorrhage. Over the years, the rates of venous thromboembolic events (VTEs) have decreased, while there seems to be an increase in the occurrence of postoperative haemorrhage. Tranexamic acid (TXA) can potentially lower the incidence of postoperative haemorrhage. This trial aims to investigate whether preoperative administration of TXA reduces the preoperative and postoperative haemorrhage rates in laparoscopic sleeve gastrectomy (LSG). Methods and analysis This is a single centre doubleblind randomised placebo-controlled trial. Patients undergoing an LSG are included after obtaining informed consent. Patients are randomised between two groups: (1) administration of placebo infusion and (2) administration of $1500 \mathrm{mg}$ TXA. In both groups, the infusions will be administered during the induction phase of the procedure. Primary outcome measures are preoperative use of haemostatic clips, postoperative haemoglobin decrease and postoperative haemorrhage. Secondary outcome measure is the rates of VTE.

Ethics and dissemination The protocol version 3 was approved by the medical ethical committee Medical Research Ethics Committees United (MEC-U), Nieuwegein, on 29 July 2019. The trial results will be submitted for publication in a peer-reviewed journal and at conference presentations.

Trial registration number The Netherlands Trial Registry (NL8029); Pre-results.

\section{BACKGROUND}

Because of the increasing rates of morbid obesity and herewith increasing incidence of bariatric surgery, the popularity of fasttrack protocols is growing. These Enhanced Recovery After Bariatric Surgery (ERABS) protocols often include short-term thromboprophylaxis and short length of hospital stay (LOS), with the aim to discharge patients on

\section{Strengths and limitations of this study}

- Randomised double-blinded placebo-controlled trial.

- Investigating a severe complication of bariatric surgery.

- Monocentre study in the Netherlands.

- Four different operators, causing inter-operator variance.

Only one dose of tranexamic acid is investigated.

the first postoperative day. ${ }^{12}$ Even though ERABS has many positive outcomes, it may also negatively affect the diagnosis of postoperative haemorrhage due to the shorttime window for diagnosing postoperative complications.

The current literature states that postoperative haemorrhage occurs in $2.0 \%$ of patients undergoing laparoscopic sleeve gastrectomy (LSG) and in $1.5 \%-3.1 \%$ of patients undergoing a laparoscopic Roux-en-Y gastric bypass (LRYGB) ${ }^{34}$ It is described that over the years, there seems to be an increase in the occurrence of postoperative haemorrhage, while the risk of venous thromboembolic events (VTEs) is decreasing. ${ }^{5}$ In our centre, overall postoperative haemorrhage rates of LRYGB and LSG have been stable since 2014, with more haemorrhage occurring after an LSG $(2.2 \%)$ than after an LRYGB $(1.3 \%)$. The rates of preoperative haemorrhage requiring haemostatic clips were $63.3 \%$ between January 2017 and March 2019. The rate of diagnosed VTE within 3 months postoperative is $0.05 \%$ since 2014 at our centre.

Tranexamic acid (TXA) is a plasminogen inhibitor that can be used to inhibit fibrinolysis during or after surgery and thus minimise the risk of developing perioperative haemorrhage. Little is known about the use of TXA 
in bariatric surgery. Nevertheless, in several other surgical areas, such as for coronary artery surgery and arthroplasty, perioperative administration of TXA has proven its value in preventing postoperative haemorrhage. ${ }^{6-8}$ Until now, the use of TXA to prevent postoperative haemorrhage in bariatric surgery has been described once by Hussain $e t$ $a l .{ }^{9}$ The authors performed a meta-analysis of administration of TXA during LSGs and concluded that administration of TXA is a simple yet effective way to lower the rates of staple line haemorrhage, while shortening the length of surgery. In the end of 2018, Klaassen et al described that reoperations may be prevented due to postoperative administration of TXA in case of a suspicion of an already present haemorrhage. ${ }^{10}$

Because of the serious risks of postoperative haemorrhage, the need for preventive measures in bariatric fasttrack surgery is increasing. This manuscript reports the protocol of a randomised controlled trial (RCT) investigating the outcome of preoperative administration of TXA in patients undergoing an LSG. The hypothesis is that the administration of TXA can lead to less use of preoperative haemostatic staples, a smaller haemoglobin decrease postoperatively and a lower reoperation rate, without increasing the VTE rates. By publishing this protocol, we hope to create more awareness of the haemorrhage risks of bariatric surgery and to further stimulate research in this area.

\section{METHODS/DESIGNS}

\section{Study design and participants}

This is a double-blind, single centre, parallel-group RCT that takes place in a high-volume bariatric centre in the Netherlands. We aim to include 100 patients. Patients are randomised with an allocation ratio of 1:1 between two groups: (1) administration of a placebo infusion (sodium chloride) and (2) administration of $1500 \mathrm{mg}$ TXA. In both groups, the infusions will be administered during the induction phase of the procedure by the anaesthesiologist.

All patients found suitable for bariatric surgery according to the international guidelines and undergoing an LSG will be asked to participate in this study. In order to be eligible to participate in this study, a subject must meet all of the following criteria: primary bariatric procedure and good knowledge of the Dutch or English language. A potential subject who meets any of the following criteria will be excluded from participation in this study: patients unwilling to give informed consent, patients with a medical history of bleeding or VTE and patients who use therapeutic anticoagulants. Patients will also be excluded in case of preoperative arterial bleeding or (iatrogenic) bleeding coming from surrounding organs or vascular structures, such as the liver or the spleen.

\section{Patient involvement}

This research was done without patient involvement. Patients were not invited to comment on the study design and will not be consulted to develop patient relevant outcomes or interpret the results. Patients will not be invited to contribute to the writing or editing of this document for readability or accuracy.

\section{Outcome measures}

Primary outcome measures are preoperative use of haemostatic staples, the decrease in haemoglobin after the procedure at day one postoperative and rates of postoperative haemorrhage (ie, haemorrhage needing administration of packed cells or a surgical or radiological re-intervention) within 30 days postoperative. Secondary outcome measure is rates of VTE within 3 months postoperative. Comorbidities such as hypertension, diabetes en dyslipidemia will be taken into account. Also, the preoperative blood pressure will be registered.

\section{Interventions}

This study will compare an intervention group with a control group. In the intervention group, patients will be administered TXA (Cyklokapron) $1500 \mathrm{mg}$ intravenously during the induction of the procedure. A set dose of $1500 \mathrm{mg}$ will be used, based on the study population with morbid obesity. ${ }^{11}$ The TXA will be intravenously administered, dissolved in $100 \mathrm{~mL}$ sodium chloride $0.9 \%$ in a time frame of $15-30 \mathrm{~min}$, with a maximum of $100 \mathrm{mg} / \mathrm{min}$. In the control group, patients will receive a placebo infusion containing $100 \mathrm{~mL}$ sodium chloride $0.9 \%$, to be administered in a time frame of $15-30 \mathrm{~min}$. The hospital's pharmacy will prepare and label the investigational medicinal products according to the relevant Good Manufacturing Practice guidelines.

For all patients participating in the trial, three topics are addressed in more detail in this protocol. First, we want to determine the haemoglobin decrease. A blood sample (one EDTA tube) will be obtained in the week preoperative by venipuncture. From this blood sample, a haemoglobin test is performed in the hospital's laboratory. The postoperative haemoglobin value will be obtained from the blood sample that is obtained in all bariatric patients on the first postoperative day. Second, we aim for an unambiguous policy on fluid balance. Patients can eat up to 8 hours preoperatively and drink clear fluids up to 4 hours preoperatively. To compensate for this preoperative 'nothing per os' policy, all patients receive $1 \mathrm{~L}$ of Ringer's lactate solution preoperatively. After the procedure, all patients receive intravenous administration of sodium chloride $0.9 \%$ ( $2 \mathrm{~L}$ per 24 hours). Furthermore, the fluid intake of each individual patient is registered starting after surgery. Third, the preoperative conditions need to be standardised for all trial patients. The preoperative mean arterial pressure is to be kept above $60 \mathrm{~mm}$ $\mathrm{Hg}$. Information on preoperative use of haemostatic staples, administration of packed cells, postoperative TXA, rates of postoperative haemorrhage and VTE are obtained from the electronical patient report. Adverse events will be reported to the medical ethical committee and the national trial committee. All patients undergo an 
Box 1 Steps to be followed by the surgeon before placement of haemostatic clips

Steps before placing haemostatic clips:

- Staple gastric sleeve.

- Remove nasogastric tube.

- Remove sleeve from the abdomen.

- Aim for normotension.

- Decrease intraabdominal pressure to $12 \mathrm{~mm} \mathrm{Hg}$.

- Inspection of staple line.

Place clips in case of $\geq 1$ bleeding spot.

LSG as described by Gadiot et al. ${ }^{12}$ Haemostatic clips will be placed according to the step-by-step plan as detailed in box 1 .

\section{Education of study personnel}

All involved personnel are educated on the study design by the coordinating researcher before the start of this study by walking through a digital presentation. In this presentation, all tasks will be discussed in detail, specified by function type. Surgeons are informed of how to include patients in this study at the outpatient clinic. The personnel of the hospital pharmacy are educated on how to prepare the individually labelled study medication. The anaesthesiology team is informed on the study design and to be aware of administering the medication as delivered by the hospital pharmacy. Bariatric nurses are educated on how to send out study information to all patients eligible for a bariatric procedure. Another important task of the bariatric nurse is the follow-up after surgery, which is done by a phone call 1 week after surgery. In this phone call, patients are asked about respiratory complaints of calve pain. In this same phone call, patients are once again reminded of the importance to contact the bariatric clinic in case of calve pain or respiratory complaints. No active follow-up (eg, duplex imaging of the calve veins) is performed to diagnose asymptomatic VTE, as it is unclear whether the not-clinically apparent VTEs are relevant to diagnose and should receive aggressive therapy when diagnosed.

\section{Sample size}

The performed power analysis (power $=80 \%, \alpha=5 \%$ twosided) calculated a required sample size of $2 \times 36=72$ patients. The analysis was based on an expected $50 \%$ decrease of the percentage of patients for whom preoperative placement of haemostatic staples was required. To be able to compensate for potential exclusions, we decided to include 100 patients in total.

\section{Recruitment}

In the Franciscus Gasthuis \& Vlietland, approximately 1000 patients undergo a bariatric procedure each year. Approximately $40 \%$ of these patients undergo a primary LSG and is herewith eligible for inclusion in this trial. Therefore, we expect to have included 100 patients within 3-6months. All patients found suitable for bariatric surgery according to the international guidelines and undergoing an LSG will have received written information attached with the invitation letter for the appointment at the outpatient clinic. The surgeon and/or the anaesthesiologist will inform the patient once more about the study protocol at the outpatient clinic. If willing to participate, patients will be asked to fill in the informed consent form. Patients will have 1 week to reconsider their decision.

\section{Blinding and randomisation}

After obtaining informed consent at the outpatient clinic of the surgical or anaesthesiological department, patients will be randomised into one of the two groups using variable block randomisation software by Ciwit BV (Castor $\left.\mathrm{EDC}^{\odot}\right)$. Patients will either receive TXA or placebo (sodium chloride) during induction. Patient, surgical team and anaesthesiological team will be blinded for the treatment, as the hospital pharmacy will prepare the infusion bags for each individual patient. Availability of the patient's individualised infusion bag will be checked at the time out procedure. The coordinating researcher and the pharmacologist are unblinded in order to prepare the infusions properly. In case of suspicion of complications due to administration of the trial infusion bag, the anaesthesiologist and the surgical team can be unblinded by contacting the pharmacy or the coordinating researcher by a phone call.

\section{Data management}

Identification of participants will be protected by using study numbers non-traceable to patient's identity. Only members of the research team will have access to the databases with the study data. Data will be kept for 20 years. The data will be monitored by a committee that is appointed by the hospital's research department.

\section{Statistical analysis}

Statistical analyses will be performed using IBM SPSS version 24 (IBM Corporation, Armonk, New York, USA). Efforts will be made to prevent missing data by checking the completeness of the collected data. Missing data may be expected because of failed haemoglobin tests or (less likely) due to unregistered administration of TXA or packed cells. Differences in use of haemostatic staples between the two groups will be calculated using $\chi^{2}$ tests. The difference in haemoglobin decrease will be tested using multivariate logistic regression analysis, correcting for comorbidities (hypertension, diabetes and dyslipidemia) and use of haemostatic staples. These same analyses will be performed to assess the differences in number of patients needing to undergo a re-intervention, but adding postoperative administration of TXA as a covariate. Differences in the rates of VTE and postoperative administration of TXA between the two groups will be calculated using $\chi^{2}$ (Fisher's exact) tests. Results will be evaluated at a significance threshold of $p<0.05$ (two-sided). 


\section{DISCUSSION}

The complication rates in a fast-track bariatric surgery are modest: $2 \%-4 \%$ of all patients undergoing a bariatric procedure develops a major complication, such as staple line leakage or haemorrhage. ${ }^{1314}$ Haemorrhage is not a frequently seen complication, but does have a substantial impact on the patient's recovery. This study tests the effects of preoperative administration of TXA on preoperative and postoperative haemorrhage rates in patients undergoing an LSG.

TXA has already been proven to be beneficial in prevention of haemorrhage in several studies. The range of the dosage of TXA among these studies is wide, varying from $100 \mathrm{mg} / \mathrm{kg}$ body weight ${ }^{8}$ to $20 \mathrm{mg} / \mathrm{kg}$ body weight, ${ }^{15}$ or multiple doses of $10 \mathrm{mg} / \mathrm{kg}$ before and after surgery. ${ }^{16}$ The dosage of TXA can be determined based on patient's body weight or renal function, or a standard dose can be given to each patient. ${ }^{17}$ In this study, a fixed dosage of $1500 \mathrm{mg}$ TXA was used. For general (non-topical) fibrinolysis in adults, TXA is administered intravenously in a dosage of $15 \mathrm{mg} / \mathrm{kg}$ body weight in a solution with sodium chloride. Administration can be repeated every $6-8$ hours. ${ }^{18}$ In our study population, body weight varies between 100 and $200 \mathrm{~kg}$. TXA has a small volume of distribution (9-12 L) and will mostly be present in a watery environment and will not spread to fatty tissue. ${ }^{11}$ Therefore, it is expected that a dosage above $1500 \mathrm{mg}$ will not be of additional value.

Very little is known about the risks of TXA in patients with an increased risk of VTE. In this study, patients who used therapeutic anticoagulants were, therefore, excluded from participation. Earlier research showed that patients who use anticoagulants are at the highest risk of developing postoperative haemorrhage. ${ }^{19}$

As administration of TXA can hypothetically increase the risk of VTE, patients will be followed up for 3 months postoperatively to diagnose symptomatic VTEs. This follow-up is observational, meaning that no routine diagnostic imaging of the calve veins will be performed. The VTE rates in a metabolic fast-track surgery are very low: $<1 \%$. Therefore, we do not expect to be able to find a significant difference in the VTE rates between the groups.

The procedures in this trial will be performed by one of the four metabolic surgeons, assisted by a surgical resident or a physician assistant. This might create a risk of inter-operator variance on placing haemostatic clips. We have, therefore, created a step-by-step plan to create uniformity in the placement of haemostatic clips. Also, the operator will be included as a covariate in the multivariate regression analysis.

In this trial, we investigate the effect of preoperative TXA in patients undergoing an LSG on the usage of haemostatic clips, haemoglobin decrease, re-interventions due to haemorrhage and the rates of VTE. Our results will be relevant for clinics performing fasttrack metabolic surgery, as administration of TXA may decrease preoperative and postoperative haemorrhage rates. We aim to present the outcome of this trial in future publications.

\section{Trial status}

The protocol version 3 was approved by the medical ethical committee on 29 July 2019. ${ }^{11}$ The enrolment of the first patient is expected in November 2019. Expected completion of recruitment is halfway through 2020.

\section{ETHICS AND DISSEMINATION}

The trial results will be submitted for publication in a peer-reviewed journal and at conference presentations.

Contributors ML was the main author of the manuscript. JH revised the pharmacological part. JMAW advised on the feasibility of the protocol. SJCV revised the anaesthesiological part. LUB and MD were important co-authors from a surgical and logistical point of view. JAA supervised the project. All authors meet the ICMJE criteria for authorship.

Funding This study was funded by the departments of surgery and pharmacology. Competing interests None declared.

Patient consent for publication Not required.

Ethics approval All procedures performed in studies involving human participants will be in accordance with the ethical standards of the institutional and/or national research committee and with the 1964 Declaration of Helsinki and its later amendments or comparable ethical standards. The study protocol was approved by the IRB and the regional MEC-U, Rotterdam, the Netherlands (protocol number R19.041).

Provenance and peer review Not commissioned; externally peer reviewed.

Data availability statement Data are available upon reasonable request. The data that will be shared is individual participant data that underlie the results reported in this article, after deidentification (text, tables, figures and appendices). Also, the study protocol will be available. Data will be available beginning 9 months and ending 36 months following publication, for researchers who provide a methodologically sound proposal for any purpose. Proposals may be directed to j. apers@franciscus.nl up to 36 months following article publication. After 36 months the data will be available in our hospital's data warehouse but without investigator support other than deposited metadata.

Open access This is an open access article distributed in accordance with the Creative Commons Attribution Non Commercial (CC BY-NC 4.0) license, which permits others to distribute, remix, adapt, build upon this work non-commercially, and license their derivative works on different terms, provided the original work is properly cited, appropriate credit is given, any changes made indicated, and the use is non-commercial. See: http://creativecommons.org/licenses/by-nc/4.0/.

ORCID iD

Marjolijn Leeman http://orcid.org/0000-0001-7747-0500

\section{REFERENCES}

1 Małczak P, Pisarska M, Piotr M, et al. Enhanced recovery after bariatric surgery: systematic review and meta-analysis. Obes Surg 2017;27:226-35.

2 Mannaerts GHH, van Mil SR, Stepaniak PS, et al. Results of implementing an enhanced recovery after bariatric surgery (ERABS) protocol. Obes Surg 2016;26:303-12.

3 Zafar SN, Miller K, Felton J, et al. Postoperative bleeding after laparoscopic Roux en Y gastric bypass: predictors and consequences. Surg Endosc 2019;33:272-280.

4 Zellmer JD, Mathiason MA, Kallies KJ, et al. Is laparoscopic sleeve gastrectomy a lower risk bariatric procedure compared with laparoscopic Roux-en-Y gastric bypass? A meta-analysis. Am J Surg 2014;208:903-10. discussion 9-10.

5 Becattini C, Agnelli G, Manina G, et al. Venous thromboembolism after laparoscopic bariatric surgery for morbid obesity: clinical burden and prevention. Surg Obes Relat Dis 2012;8:108-15. 
6 Kirsch JM, Bedi A, Horner N, et al. Tranexamic acid in shoulder arthroplasty: a systematic review and meta-analysis. JBJS Rev 2017;5:e3.

7 Meftah M, White PB, Siddiqi A, et al. Tranexamic acid reduces transfusion rates in obese patients undergoing total joint arthroplasty. Surg Technol Int 2019;34:451-455.

8 Myles PS, Smith JA, Forbes A, et al. Tranexamic acid in patients undergoing coronary-artery surgery. N Engl J Med 2017;376:136-48.

9 Hussain A, Al-Shoek I, El-Hasani S. The use of tranexamic acid in sleeve gastrectomy. Obes Surg 2017;27:198-9.

10 Klaassen RA, Selles CA, van den Berg JW, et al. Tranexamic acid therapy for postoperative bleeding after bariatric surgery. BMC Obes 2018;5:36.

11 National Center for Biotechnology Information. PubChem database. tranexamic acid, CID=5526. Available: https://pubchem.ncbi.nlm.nih gov/compound/Tranexamic-acid [Accessed 29 Jul 2019].

12 Gadiot RPM, Biter LU, Zengerink HJF, et al. Laparoscopic sleeve gastrectomy with an extensive posterior mobilization: technique and preliminary results. Obes Surg 2012;22:320-9.

13 Blanchet M-C, Gignoux B, Matussière $\mathrm{Y}$, et al. Experience with an enhanced recovery after surgery (ERAS) program for bariatric surgery: comparison of MGB and Isg in 374 patients. Obes Surg 2017;27:1896-900

14 Dogan K, Kraaij L, Aarts EO, et al. Fast-Track bariatric surgery improves perioperative care and logistics compared to conventional care. Obes Surg 2015;25:28-35.

15 Friedman RJ, Gordon E, Butler RB, et al. Tranexamic acid decreases blood loss after total shoulder arthroplasty. J Shoulder Elbow Surg 2016;25:614-8

16 Pauzenberger L, Domej MA, Heuberer PR, et al. The effect of intravenous tranexamic acid on blood loss and early postoperative pain in total shoulder arthroplasty. Bone Joint J 2017;99B:1073-9.

17 Melvin JS, Stryker LS, Sierra RJ. Tranexamic acid in hip and knee arthroplasty. J Am Acad Orthop Surg 2015;23:732-40.

18 CBG. Samenvatting van de productkenmerken Tranexaminezuur Mylan 100 mg/ml, oplossing voor injectie. Geneesmiddeleninformatiebank, 2017.

19 Coblijn UK, Karres J, de Raaff CAL, et al. Predicting postoperative complications after bariatric surgery: the bariatric surgery index for complications, basic. Surg Endosc 2017;31:4438-45. 